\title{
Cardiovascular Protective Actions of Sodium Glucose Cotransporter 2 Inhibitors
}

\section{Shamanna S lyengar}

"Current management of diabetes is characterized by opportunities, options, and obstacles."

-Dr Sanjay Kalra

\begin{abstract}
Diabetes mellitus is a major health problem in our country, and the prevalence is on the increase. Cardiovascular complications are the commonest causes of mortality and morbidity in patients with type II diabetes mellitus. Diabetes may not be a coronary artery disease (CAD) equivalent, but it certainly carries a high risk for atherosclerotic cardiovascular disease (CVD). There are effective drugs to treat hyperglycemia, and these drugs may be having adverse effects or advantageous outcomes on CVD, or they may be neutral. While developing antidiabetic drugs, it has become necessary to study their effect on CVD and outcome.

Among the newer antidiabetic drugs, sodium glucose cotransporter 2 (SGLT2) inhibitors have exhibited impressive cardiovascular benefits. Various mechanisms have been proposed to explain their improved cardiovascular outcome. They are not without adverse effects. There are a number of SGLT2 inhibitor preparations, and it is debatable whether this cardiovascular benefit is a class effect or individual drug specific. These newer antidiabetic drugs are looking beyond blood sugar control.
\end{abstract}

Keywords: Cardioprotective antidiabetic drugs, Heart and diabetes, Sodium glucose cotransporter 2 inhibitors.

How to cite this article: lyengar SS. Cardiovascular Protective Actions of Sodium Glucose Cotransporter 2 Inhibitors. Hypertens J 2017;3(3):161-165.

Source of support: Nil

Conflict of interest: None

\section{INTRODUCTION}

In a highly informative article with a catchy phrase embedded in the title, "Nations within a nation," providing estimates of 333 diseases and 84 risk factors from different states of India, covering the period from 1990 to 2016, it has been reported that CVD and diabetes accounted for 15.9 and $8.9 \%$ of the disability-adjusted life years respectively. ${ }^{1}$

Consultant

Department of Cardiology, Manipal Hospitals, Bengaluru Karnataka, India

Corresponding Author: Shamanna S lyengar, Consultant Department of Cardiology, Manipal Hospitals, Bengaluru Karnataka, India, e-mail: ssiyengar1945@gmail.com
Diabetes is highly prevalent and is potentially an epidemic in India with more than 62 million individuals diagnosed to have type II diabetes. ${ }^{2,3}$

Diabetic state poses serious problems with microvascular and macrovascular complications. There is a strong relationship between CVD and diabetes, CVD accounting for most of the mortality and morbidity among type II diabetic patients. Adults with diabetes are two to four times more likely to die from heart disease than adults without diabetes. At least $68 \%$ of the diabetic people in the age group 65 years or older die of heart disease and $16 \%$ die of stroke. ${ }^{4}$ Haffner et $\mathrm{l}^{5}$ stated that patients with type II diabetes without a previous history of myocardial infarction have the same risk of CAD as nondiabetic subjects with a history of myocardial infarction. However, this claim has been challenged by subsequent studies. A systematic review and metaanalysis showed that patients with diabetes without prior myocardial infarction have a $43 \%$ lower risk of CAD as compared with patients without diabetes with previous myocardial infarction. ${ }^{6}$ A population-based prospective cohort analysis concluded that all diabetics should not be considered to be at a risk equivalent to that of patients having prior CAD. ${ }^{7}$

The increased risk of CVD in diabetics is thought to be due to hyperglycemia and the consequent intracellular metabolic changes resulting in oxidative stress, low-grade inflammation, and endothelial dysfunction. Generally, diabetics have associated comorbidities like obesity, hypertension, and dyslipidemia, which contribute to an increased risk for CVD. ${ }^{8}$

The benefit of lowering blood sugar levels in diabetics has been well established in the UK Prospective Diabetes Study trial. ${ }^{9}$ With a number of antidiabetic drugs available now, it has become necessary that cardiologists get themselves familiar with antidiabetic drug classes that have cardiovascular benefits. ${ }^{10}$

In view of this complex issue of diabetic treatment and cardiovascular risk, regulatory agencies have made it mandatory to produce evidence based on adjudicated outcomes from clinical trials that there is cardiovascular benefit or that the risk of cardiovascular harm is low for all new glucose-lowering therapies before a drug is made available, and usually also require a postmarketing cardiovascular safety trial. 


\section{SODIUM GLUCOSE COTRANSPORTER 2 INHIBITORS}

The SGLT2 inhibitors have brought in some encouraging news, brightening up the field of the depressive reports of antidiabetic drugs in the past. Other drugs that have shown cardiovascular benefits are metformin, pioglitazone, and glucagon-like peptide-1 receptor agonists. The SGLT2 inhibitors are unique antidiabetic drugs, and by inhibiting SGLT2 in the proximal convoluted tubule prevent re-absorption of glucose and facilitate its excretion in urine. As glucose is excreted, its plasma levels fall leading to an improvement in all glycemic parameters, and this action is independent of the actions of insulin. Thus, the hypoglycemia risk is minimal.

Other effects of SGLT2 inhibitors are as follows ${ }^{11-16}$ (Fig. 1):

- Sodium glucose cotransporter 2 inhibitor usage results in a reduction of body weight, and it is usually the visceral fat that is lost.

- Sodium glucose cotransporter 2 inhibitors reduce both systolic and diastolic blood pressure.

- Sodium glucose cotransporter 2 inhibitors increase high-density lipoprotein (HDL) by about 7 to $10 \%$, low-density lipoprotein (LDL) by $7 \%$, and reduce triglycerides by $2 \%$.

- Uric acid levels are reduced by SGLT2 inhibitors.

- Sodium glucose cotransporter 2 is one of the determinants of glomerular hyperfiltration and SGLT2 inhibition offers nephroprotective action.

\section{CLINICAL TRIALS OF SGLT2 INHIBITORS EMPA-REG Outcome ${ }^{17}$}

In this study, 7,020 diabetic patients were randomized to receive 10 or $25 \mathrm{mg}$ of empagliflozin or placebo once daily and were followed up for 3.1 years, for primary outcome of composite of death from cardiovascular causes, nonfatal myocardial infarction, or nonfatal stroke, and secondary outcome of primary outcome plus hospitalization for heart failure.

More than $90 \%$ of patients had established CVD [myocardial infarction, cerebrovascular accident, peripheral arterial disease (PAD)], and they were on appropriate treatment for hypertension and dyslipidemia.

There was a statistically significant $14 \%$ reduction in primary endpoint, $38 \%$ reduction in cardiovascular death, $35 \%$ reduction in hospitalization for heart failure, and $32 \%$ reduction in death from any cause. The difference between empagliflozin and placebo was mainly driven by a significant reduction in death from cardiovascular causes, there being no significant difference in the risk of myocardial infarction or stroke. The cardiovascular benefits were seen early in the trial, and continued throughout.

One notable adverse event was an increased rate of genital infection with empagliflozin.

Empagliflozin reduced $\mathrm{HbA1c}$ significantly and was also seen to result in small reductions in body weight, waist circumference, uric acid level, and systolic and diastolic blood pressure with no increase in heart rate and small increases in both LDL and HDL cholesterol. The investigators of this study are of the opinion that cardiovascular benefits of empagliflozin are multidimensional. Apart from their effects on blood sugar, body weight, central adiposity, and blood pressure, they probably induce favorable changes in arterial stiffness, cardiac function, cardiac oxygen demand, cardiorenal effects, reduction in albuminuria, and reduction in uric acid.

\section{EMPA-REG Outcome Substudy ${ }^{18}$}

In this substudy, 982 patients who had PAD treated with empagliflozin were compared with 479 patients of PAD receiving placebo. Those receiving empagliflozin had significant reduction in mortality, hospitalization for heart failure, and progression of renal disease and there was no increase in the risk of lower limb amputation.

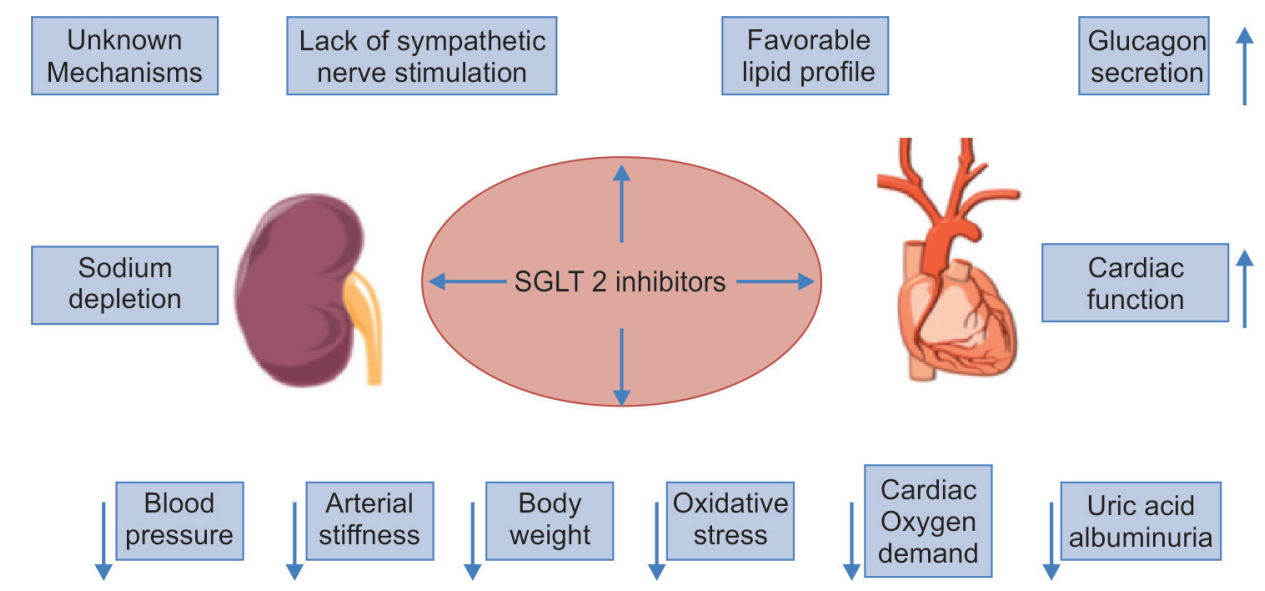

Fig. 1: Possible mechanisms to explain CV benefits of SGLT2 inhibitors (Modified from Inzucchi et al. Diabetes Vasc Dis Res 2015;12:90-100) 


\section{CANVAS Trial ${ }^{19}$}

This study had 10,142 participants with type II diabetes and high cardiovascular risk. Nearly $65 \%$ of them had CVD and were randomized to receive canagliflozin or placebo. They were followed up for a mean of 188.2 weeks. The primary outcome was a composite of death from cardiovascular causes, nonfatal myocardial infarction, or nonfatal stroke. Secondary outcomes studied were death from any cause, death from cardiovascular causes, progression of albuminuria, and the composite of death from cardiovascular causes and hospitalization for heart failure. The primary endpoint was reduced by a significant $14 \%$ in the canagliflozin arm. It also reduced the risk of the progression of albuminuria, the need for renal-replacement therapy, and death from renal causes. The points of concern were an increased risk of amputation, though primarily of toe or metatatrsal, and an increased risk of fractures.

The authors proposed that improved glycemic control, lowering of blood pressure, decrease in intraglomerular pressure, reduction in albuminuria, and amelioration of volume overload offered by canagliflozin are probably the mechanisms responsible for cardiovascular and renal protection.

\section{Results from the CANVAS Program ${ }^{20}$}

In this study, the efficacy and safety of canagliflozin were analyzed separately for the primary and secondary prevention cohorts enrolled in the CANVAS Program. There were 3,486 primary prevention participants and 6,658 secondary prevention participants. Canagliflozin reduced cardiovascular and renal outcomes in both the primary and secondary prevention groups, though authors cautioned about the use of canagliflozin in patients at risk for amputations.

\section{Dapagliflozin ${ }^{21}$}

Dapagliflozin, like other SGLT2 inhibitors, has low risk for hypoglycemia, lowers blood pressure, decreases weight and waist circumference, and decreases albuminuria and serum uric acid levels.

A meta-analysis of 9,339 patients with type II diabetes and increased cardiovascular risk and received dapagliflozin had the following results. Overall population had a $23 \%$ lower risk of major adverse cardiovascular event (MACE) and patients with history of CVD had 20\% lower risk of MACE.

\section{Meta-analysis of SGLT2 Inhibitors ${ }^{22}$}

In a meta-analysis of 37,525 patients who received one of the seven SGLT2 inhibitors (canagliflozin, dapagliflozin, empagliflozin, ipragliflozin, luseogliflozin, tofogliflozin, and ertugliflozin), the study looked at the primary outcome of MACE: CV death, nonfatal myocardial infarction, and nonfatal stroke.

The key conclusions were:

- Sodium glucose cotransporter 2 inhibitors significantly decreased MACE, cardiovascular death, and all-cause mortality.

- The risk of heart failure also decreased, but data were available only for empagliflozin.

- Sodium glucose cotransporter 2 inhibitor use was linked to significantly increased risk of nonfatal stroke.

- Cardiovascular results were driven largely by outcomes from empagliflozin reported from a single study, and more studies are needed to confirm these findings across the drug class

\section{Sodium Glucose Cotransporter 2 Inhibitors- Class Effect?}

To date, three such drugs - canagliflozin, dapagliflozin, and empagliflozin — have been approved by international regulatory agencies for the treatment of type II diabetes. Impact on hospitalization for heart failure was similar in both CANVAS (with canagliflozin) and EMPA-REG OUTCOME (with empagliflozin) trials. This suggests their similarity in hemodynamic effects.

If one looks at the individual components of end points in the two trials, there are some differences. There was an increase in amputations in the CANVAS trial with canagliflozin, which is not fully explained, and it is noted that the European Medicines Agency has urged caution to be exercised for the class (in particular canagliflozin) in patients with previous foot complications. In EMPA-REG OUTCOME (with empagliflozin) trial, there was a statistically nonsignificant increase in nonfatal stroke rate.

The SGLT2 inhibitors do share some common features, but there could be some minor differences. Ongoing trials will probably clarify the picture.

\section{SUMMARY}

The effects of SGLT2 may be summarized as below:

\section{Adverse Effects}

- Urogenital tract infections are the most frequently noticed adverse events in subjects on SGLT2 inhibitors.

- The osmotic diuresis associated with SGLT2 inhibitor use also has the potential to cause volume depletion and orthostatic hypotension.

- The SGLT2 inhibitor use leads to a slight reduction in bone formation, and a rise in bone resorption markers.

- The SGLT2 inhibitors should not be used in patients with type I diabetes where its use is limited to clinical research studies. The Food and Drug Administration issued a warning about the risk of ketoacidosis 
occurring in the absence of significant hyperglycemia (euglycemic diabetic ketoacidosis) in patients with types I and II diabetes treated with SGLT2 inhibitors.

\section{Glucose Lowering Effect}

The SGLT2 inhibition in patients on insulin regimens results in further $\mathrm{HbA1c}$ reduction, lower insulin dose requirement, and greater weight loss, and there is no worsening of hypoglycemia. The SGLT 2 inhibitors usually reduce $\mathrm{HbA} 1 \mathrm{c}$ by 0.7 to $1.0 \%$.

\section{Nonglycemic Effects of SGLT2 Inhibitor ${ }^{23}$}

- Elevations in HDL cholesterol and reduction in triglyceride concentrations along with a minor elevation of LDL cholesterol should not cause concern over CVD risk. The EMPA-REG OUTCOME trial has refuted this concern, along with the fact that SGLT2 inhibitor switches metabolism from carbohydrate toward lipid utilization, which moderately increases ketogenesis and low-density lipoprotein concentrations despite net lipid metabolic utilization.

- Blood pressure lowering: several factors are likely to be involved including changes in plasma volume and reduced arterial stiffness.

- Reduction in body weight induced by SGLT2 inhibitors: the effect is seen mainly on visceral obesity. The SGLT2 inhibitors have neutral effects on the sympathetic nervous system. The SGLT2 inhibition does not increase heart rate despite the plasma volume reduction associated with the class.

- The SGLT2 inhibitor-mediated reductions in hyperfiltration would suppress markers of inflammation and fibrosis.

- Dapagliflozin has been shown to reduce C-reactive protein.

- Renal hemodynamic function and albuminuria: SGLT2 inhibitors reduce albuminuria by 30 to $40 \%$, possibly based on reductions in intraglomerular hypertension.

- Uric acid: The SGLT2 inhibitor class of drugs has been associated with a 10 to $15 \%$ reduction in plasma uric acid levels.

Beneficial effects seem to outweigh a small number of adverse actions of SGLT2 inhibitors. The glycemic and nonglycemic effects of SGLT2 inhibitors enumerated above probably explain the cardiovascular benefits imparted by these agents.

\section{RECOMMENDATIONS}

The American Diabetes Association ${ }^{24}$ has recommended the following:

"In patients with long-standing suboptimally controlled type II diabetes and established atherosclerotic
CVD, empagliflozin or liraglutide should be considered as they have been shown to reduce cardiovascular and all-cause mortality when added to standard care. Ongoing studies are investigating the cardiovascular benefits of other agents in these drug classes."

\section{CONCLUSION}

Now that there are antidiabetic agents that offer glycemic control and, in addition, reduce cardiovascular events, the physician or endocrinologist or cardiologist has a responsibility to consider the use of these and avoid drugs that are likely to harm or offer no benefit.

It is probably appropriate to conclude

"Current management of diabetes is characterized by opportunities and options, and not obstacles."

\section{REFERENCES}

1. India State-level Disease Burden Initiative Collaborators. Nations within a nation: variations in epidemiological transition across the states of India, 1990-2016 in the Global Burden of Disease Study. Lancet 2017 Dec;390(10111):2437-2460.

2. Joshi SR, Parikh RM. India - diabetes capital of the world: now heading towards hypertension. J Assoc Physicians India 2007 May;55:323-324.

3. Kumar A, Goel MK, Jain RB, Khanna P, Chaudhary V. India towards diabetes control: key issues. Australas Med J 2013 Oct; 6(10):524-531.

4. Centers for Disease Control and Prevention. National diabetes fact sheet: national estimates and general information on diabetes and prediabetes in the United States. Atlanta (GA): CDC; 2011.

5. Haffner SM, Lehto S, Ronnemaa T, Pyörälä K, Laakso M. Mortality from coronary heart disease in subjects with type 2 diabetes and in nondiabetic subjects with and without prior myocardial infarction. N Engl J Med 1998 Jul;339(4): 229-234.

6. Bulugahapitiya U, Siyambalapitiya S, Sithole J, Idris I. Is diabetes a coronary risk equivalent? Systematic review and meta-analysis. Diabet Med 2009 Feb;26(2):142-148.

7. Rana JS, Liu JY, Moffet HH, Jaffe M, Karter AJ. Diabetes and prior coronary heart disease are not necessarily risk equivalent for future coronary heart disease events. J Gen Intern Med 2016 Apr;31(4):387-393.

8. AlGhatrif M, Kuo YF, AlSnih S, Raji MA, Ray LA, Markides KS. Trends in hypertension prevalence, awareness, treatment and control in older Mexican Americans, 1993-2005. Ann Epidemiol 2011 Jan;21(1):15-25.

9. UKPDS. Intensive blood-glucose control with sulphonylureas or insulin compared with conventional treatment and risk of complications in patients with type 2 diabetes (UKPDS 33). UK Prospective Diabetes Study (UKPDS) Group. Lancet 1998 Sep;352(9131):837-853.

10. Sattar N, Petrie MC, Zinman B, Januzzi JL Jr. Novel diabetes drugs and the cardiovascular specialist. J Am Coll Cardiol 2017 May;69(21):2646-2656.

11. Ferrannini E, DeFronzo RA. Impact of glucose-lowering drugs on cardiovascular disease in type 2 diabetes. Euro Heart J 2015 Sep;36(34):2288-2296. 
12. Scheen AJ. Pharmacokinetic and pharmacodynamic profile of empagliflozin, a sodium glucose cotransporter 2 inhibitor. Clin Pharmacokinet 2014 Mar;53(3):213-225.

13. Tikkanen I, Narko K, Zeller C, Green A, Salsali A, Broedl UC, Woerle HJ. Empagliflozin improves blood pressure in patients with type 2 diabetes (T2DM) and hypertension. Diabetologia 2013 Nov;56(Suppl 1):S377.

14. Leiter LA, Yoon KH, Arias P, Langslet G, Xie J, Balis DA, Millington D, Vercruysse F, Canovatchel W, Meininger G. Canagliflozin provides durable glycemic improvements and body weight reduction over 104 weeks versus glimepiride in patients with type 2 diabetes on metformin: a randomized, double-blind, phase 3 study. Diabetes Care 2015 Mar;38(3):355-364.

15. ShaS, DevineniD, Ghosh A,PolidoriD,HompeschM,ArnoldsS, Morrow L, Spitzer H, Demarest K, Rothenberg P. Pharmacodynamic effects of canagliflozin, a sodium glucose co-transporter 2 inhibitor, from a randomized study in patients with type 2 diabetes. PLoS One 2014 Aug;9(8):e105638.

16. Bolinder J, Ljunggren Ö, Johansson L, Wilding J, Langkilde AM, Sjöström CD, Sugg J, Parikh S. Dapagliflozin maintains glycaemic control while reducing weight and body fat mass over 2 years in patients with type 2 diabetes mellitus inadequately controlled on metformin. Diabetes Obes Metab 2014 Feb;16(2):159-169.

17. Zinman B, Wanner C, Lachin JM, Fitchett D, Bluhmki E, Hantel S, Mattheus M, Devins T, Johansen OE, Woerle HJ, et al. Empagliflozin, cardiovascular outcomes, and mortality in type 2 diabetes. N Engl J Med 2015 Nov;373(22):2117-2128.

18. Verma S, David Mazor C, Al-Omran M, InzucchiSE, Fitchett D, Hehnke U, George JT, Zinman B. Cardiovascular outcomes and safety of empagliflozin in patients with type 2 diabetes mellitus and peripheral artery disease a subanalysis of EMPA-REG OUTCOME. Circulation 2017 Nov;136:032031.

19. Neal B, Perkovic V, Mahaffey KW, de Zeeuw D, Fulcher G, Erondu N, Shaw W, Law G, Desai M, Matthews DR; CANVAS Program Collaborative Group. Canagliflozin and cardiovascular and renal events in type 2 diabetes. N Engl J Med 2017 Aug;377(7):644-657.

20. Mahaffey KW, Neal B, Perkovic V, de Zeeuw D, Fulcher G, Erondu N, Shaw W, Sun T, Desai M, Matthews DR. Canagliflozin for primary and secondary prevention of cardiovascular events results from the CANVAS Program (Canagliflozin Cardiovascular Assessment Study). Circulation 2017 Nov;137:032038.

21. Sonesson C, Johansson PA, Johnsson E, Gause-Nilsson I. Cardiovascular effects of dapagliflozin in patients with type 2 diabetes and different risk categories: a meta-analysis. Cardiovasc Diabetol 2016 Feb;15:37.

22. Wu JH, Foote C, Blomster J, Toyama T, Perkovic V, Sundstrom J, Neal B. Effects of sodium-glucose cotransporter-2 inhibitors on cardiovascular events, death, and major safety outcomes in adults with type 2 diabetes: a systematic review and metaanalysis. Lancet Diabetes Endocrinol 2016 May;4(5):411-419.

23. Heerspink HJ, Perkins BA, Fitchett DH, Husain M, Cherney DZ. Sodium glucose cotransporter 2 inhibitors in the treatment of diabetes mellitus cardiovascular and kidney effects, potential mechanisms, and clinical applications. Circulation 2016 Sep;134(10):752-772.

24. American Diabetes Association. Pharmacologic approaches to glycemic treatment. Diabetes Care 2017 Jan;40(Suppl 1): S64-S74. 